\title{
V. Induction-balance and experimental researches therewith
}

\section{Prof. D.E. Hughes}

To cite this article: Prof. D.E. Hughes (1879) V. Induction-balance and experimental researches therewith, Philosophical Magazine Series 5, 8:46, 50-56, DOI: 10.1080/14786447908639649

To link to this article: http://dx.doi.org/10.1080/14786447908639649

曲 Published online: 13 May 2009.

Submit your article to this journal $\pi$

Џ Article views: 6

Q View related articles $\sqsubset$ 
rapid vibratory diaphragm of the telephone and phonograph of Professor Bell and Mr. Edison. For just as the receiving diaphragm, from the non-preponderating character of its very rapid natural vibrations, is able to produce truthfully in miniature the varied sound-vibrations communicated to the transmitting diaphragm, so does our seismograph trut/hfully record in miniature the earthquake shocks it has received.

And we are led to think that, if rapidity of vibration be combined with viscous resistance (as explained in the paper) telephones and phonographs may be successfully constructed of far larger dimensions and of far greater power than has hitherto been attempted.

V. Induction-balance and Experimental Researches therewith. $B y$ Prof. D. E. Hughes *.

[Plate V.]

TMMEDIATELY upon the announcement of Arago's dis1 covery of the influence of rotating plates of metal upon a magnetic needle (1824), and Faraday's important discovery of voltaic and magneto-induction (1831), it became evident that the induced currents circulating in a metallic mass might be so acted upon either by voltaic or induced currents circulating in a matallic mass as to bring some new light to bear on the molecular construction of metallic bodies.

The question was particularly studied by Babbage, Sir John Herschell, and M. Dove, who constructed an induction-balance, wherein two separate induction-coils, each having its primary and secondary coils, were joined together in such a manner that the induced current in one coil was made to neutralize the induced current in the opposite coil, thus forming an induction-balance, to which he gave the name of "differential inductors." In those days physicists did not possess the exquisitely sensitive galvanometers and other means of research that we possess today; but sufficiently important results were obtained to prove that a vast field of research would be opened if a perfect induction-balance could be found, together with a means of correctly estimating the results obtained. In experimenting with the microphone I had ample occasion to appreciate the exquisite sensitiveness of the telephone to minute induced currents. This led me to study the question of induction by aid of the telephone and microphone: the results of those researches have already been published. Continuing this line of inquiry, I thought I might again attempt to investigate the molecular construction of metals and alloys; and with this object I have obtained, after numerous compara-

* Communicated by the Physical Society. 
tive failures, a perfect induction-balance which is not only exquisitely sensitive and exact, but allows us to obtain direct comparative measures of the force or disturbances produced by the introduction of any metal or conductor.

The instrument now submitted to the Physical Society consists, 1st, of the new induction-currents balance; 2nd, a microphone, with a clock as a source of sound; 3rd, electric sonometer, or absolute sound-measurer, a late invention of my own ; 4th, a receiving telephone and thres elements of Daniell's battery. In order to have a perfect induction-currents balance suitable for physical research, all its coils as well as the size and amount of wire should be equal. The primary coils $a, a^{\prime}$, and the secondary coils $b, b^{\prime}$, should be separated and not superposed. The exterior diameter of the coils is $5 \frac{1}{2}$ centims., having an interior vacant circular space of $\frac{3}{3}$ centims.; the depth of this flat coil or spool is 10 millims. Upon this box-wood spool are wound 100 metres of No. 32 silk-covered copper wire. I use four of such coils, formed into two pairs, the secondary coil being fixed permanently, or by means of an adjustable slide, at a distance of 5 millims. from its primary ; on the second similar pair there is a fine micrometer-screw, allowing me to adjust the balance to the degree of perfection required. These two pairs of coils should be placed at a distance not less than one metre from each other, so that no disturbing cause may exist from their proximity. The two primary coils are joined in series to the battery, the circuit also passing through the microphone. In place of the telephone I have sometimes used a magnetic pendulum, the swing or the arc described indicating and measuring the forces. I am at present engaged upon a very sensitive voltameter, which shall indicate and measure the force of rigid induced currents. The telephone, however, is well adapted as an indicator, but not as a measure of the forces brought into action. For this reason I have joined to this instrument an instrument to which I have given the name of electric sonometer (Pl. V.) This consists of three coils $c, d, e$, similar to those already deseribed, two of which are placed horizontally at a fixed distance of about 40 centims. apart ; and the communication with battery is so arranged that there are similar but opposing poles in each coil ; between these two there is a coil, $d$, which can be moved on a marked sliding scale, $f$, divided into millimetres, in a line with these two opposing primary coils*. This central coil is the secondary one, and connected by means of a switching key with the telephone in place of the induc-

* If the coils $c$ and $e$ are of unequal size, the zero of the scale will occupy a position similar to that shown on the Plate.

E 2 
tion-balances. If this secondary coil is near either primary coil, we hear loud tones, due to its proximity. The same effect takes place if the secondary coil is near the opposing coil, except that the induced current is now in a contrary direction, as a similar pole of the primary acts now on the opposite side of the induction-coil. The consequence is, that as we withdraw it from one coil and approach the other, we must pass a line of absolute zero, where no current whatever can be induced, owing to the absolute equal forces acting equally on both sides of the induction-coil. This point is in the exact centre between the two coils.

We thus possess a sonometer having an absolute zero of sound : each degree that it is moved is accompanied by its relative degrees of increase; and this measure may be expressed in the degrees of the millimetres passed through, or by the square of the distances in accordance with the curve of electro-magnetic action. If we place in the coils of the induction-balance a piece of metal (say copper, bismuth, or iron), we at once produce a disturbance of the balance, and it will give out sounds more or less intense on the telephone according to the mass, or if of similar sizes, according to the molecular structure of the metal. The volume and intensity of sound is invariably the same for a similar metal. If by means of the switching key the telephone is instantly transferred to the sonometer, and if its coil be at zero, we hear sounds when the key is up, or in connexion with the wire $g$, which leads to the induction-balance, and no sounds, or silence, when the key is down or in contact with the wire $h$, in connexion with the sonometer. If the sonometer-coil were moved through several degrees, or through more than the required amount, we should find that the sounds increase when the key is depressed; but when the coil is moved to a degree where there is absolute equality, if key is up or down, then the degree on scale should give the true value of the disturbance produced in the induction-balance; and this is so exact that if we put, say, a silver coin whose value is $115^{\circ}$, no other degree will produce equality. Once knowing, therefore, the value of any metal or alloy, it is not necessary to know in advance what the metal is ; for if its equality is $115^{\circ}$, it is silver coin ; if 52 , iron ; if 40 , lead ; if 10 , bismuth; and as there is a very wide limit between each metal, the reading of the value of each is very rapid, a few seconds sufficing to give the exact sound-value of any metal or alloy.

The respective values of the different metals may, as I have already pointed out, be indicated by introducing the sonometer into circuit. I find, however, that it is difficult to estimate fractional differences when the sounds to be com- 
pared are loud. I therefore prefer to balance the metal under examination by means of a similar mass placed in the opposing coil, reading on the sonometer the differences of sound, which are then slight. Experience has shown that the most accurate results are obtained when the sonometer is replaced by a graduated strip of zinc about 23 millims. wide, 200 millims. long, and tapering from a thickness of 4 millims. at one end to a fine edge at the other, and superposed in a horizontal plane over the opposing coil $b^{\prime}$, the metal to be tested being in a plane midway between $a$ and $b$ on the left of the plate.

The delicacy of the readings may of course be greatly increased by diminishing the angle botween the two faces of the strip; but there are many points connected with its use which would be too long to describe in this paper.

As a rule three I)aniell elements will be found quite sufficient; and even this weak force is so exquisitely sensitive that it will find out the smallest fraction of difference in weight or structure of metals. Thus two silver coins such as a shilling, both quite new, and both apparently of the same weight, will be found to possess a difference of weight which the instrument at once indicates.

The following experiments will show its exceeding sensitiveness and its wide field of usefulness as an instrument of research.

I. If we introduce into one pair of the induction-coils any conducting body, such as silver, copper, iron, \&c., there are set up in these bodies electric currents which react both upon the primary and secondary coils, producing extra currents whose force will be proportional to the mass and to its specific conducting-power. A miligramme of copper or a fine iron wire, finer than the human hair, can be loudly heard and appreciated by direct measurement, and its exact value ascertained. We can thus weigh to an almost infinitesimal degree the mass of the metal under examination : for instance, if we take two English shilling pieces fresh from the Mint, and if they are absolutely identical in form, weight, and material, they will be completely balanced by placing one each in the two separate coils, provided that for these experiments there is an adjustable resting-place in each pair of coils, so that each coin may lie exactly in the centre of the vacant space between the primary and secondary coils. If, however, these shillings are in the slightest degree worn, or have a different temperature, we at once perceive this difference, and, if desired, measure it by the sonometer, or by lifting the supposed heaviest coin at a slight distance from the fixed centre line: the amount of degrees that the heaviest coin is withdrawn will show its relative mass or weight as compared with the lightest. I have thus been able to appreciate the difference 
caused by simply rubbing the shilling between the fingers, or the difference of temperature by simply breathing near the coils; and in order to reduce this sensibility within reasonable limits, I have only used in the following experiments 100 metres of copper wire to each coil, and 3 cells of battery.

II. The comparative disturbing value of disks of different metals, all of the same size and form of an English shilling, and measured in millimetre-degrees, by the sonometer, is the following:-

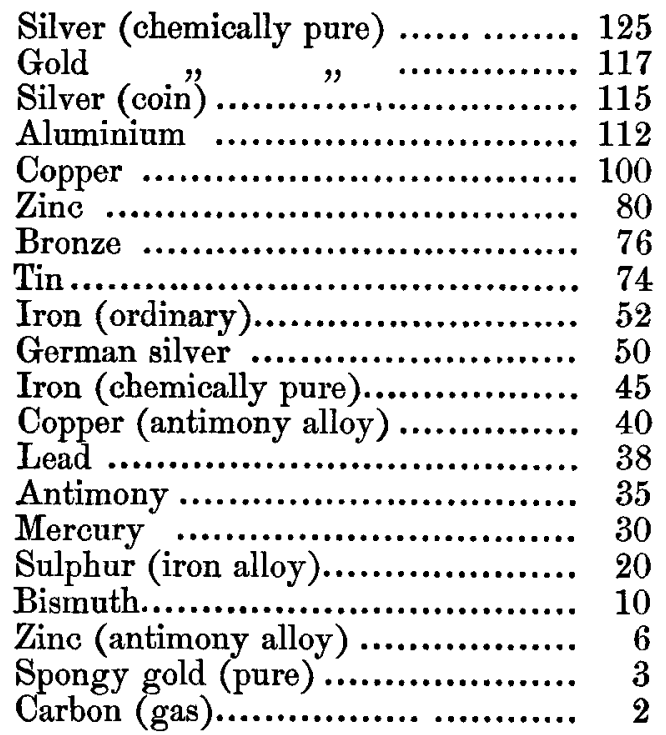

III. It will be seen from the above that the instrument gives very different values for different metals or alloys ; consequently we cannot obtain a balance by employing two disks of different metals, and the instrument is so sensitive to any variation in mass or matter that it instantly detects the difference by clear loud tones on the telephone. If I place two gold sovereigns of equal weight and value, one in each coil, there is complete silence, indicating identity or equality between them ; but if one of them is a false sovereign, or even gold of a different alloy, the fact is instantly detected by the electrical balance being disturbed. The instrument thus becomes a rapid and perfect coin-detector, and can test any alloy, giving instantly its electrical value. The exceeding sensitiveness of this electrical test I shall demonstrate by experiment, now. Again, as regards coins, it resolves an almost magical problem. Thus, if a person puts one or several coins into one pair of coils, the amount or nominal value being un- 
known to myself, I have only to introduce into the opposite coils different coins successively, as I should weights in a scale, and when perfect balance is announced by the silence, the amount in one box will not only be the same nominal value but of the same kind of coin.

IV. We find, by direct experiment with this instrument, that the preceding results are due to electric currents induced by the primary coil, and that it is by the reaction of these that the balance is destroyed; for if we take an insulated spiral disk or helix of copper wire with its terminal wires open, there is no disturbance of the balance whatever, notwithstanding that we have introduced a comparatively large amount of copper wire; but on closing the circuit the balance is at once very powerfully disturbed.

If the spiral is a flat one, resembling a disk of metal, and circuit closed, we find that loud tones result when the spiral is placed flat, or when its wire is parallel to those on the coils; but if it is held at right angles to these wires, no sound whatever is heard, and the balance remains perfect. The same thing occurs with disks of all non-magnetic metals, and a disk of metal placed perpendicular to the coils exerts no influence whatever. The contrary result takes place with a spiral of iron wire or disk of iron: the induced current circulating in the spiral is at its maximum when the spiral lies flat or parallel with the coils, giving no induced current whatever when at right angles; but the disturbances of the induction-balance are more than fourfold when perpendicular to the wires of the coils than when parallel with the same. This result is simply due to the property of magnetic bodies, of conduction of magnetism.

$V$. If we introduce a disk of metal gradually into the coils, we find that its power increases as the square of the distance, until it arrives at its maximum exactly in the centre of the vacant space between each pair of coils, diminishing rapidly in the same ratio if the disk be moved towards primary or secondary. Thus, in the interior of the coils there is but a single line of maximum force; but at the exterior we have on each coil two maxima and one minimum, the first and most powerful maximum being in the centre of vacant space between each pair of coils, coinciding with the maximum lines of force of the centre of the coils; the minimum lines of force are exactly in the exterior centre of each coil, again rapidly rising to a maximum near the exterior edge of coils, and gradually diminishing in power from this point.

If we place exteriorly a bar of metal in the centre line of vacant space, we find that it has here its maximum disturbing power, giving out loud sounds. 
If we now move this bar until it rests on the centre of either coil, we find that at this point the bar has no disturbing effect whatever, and although the coils at its maximum line of force are sensitive to the finest iron wire, a very large mass of iron or a rod of 1 centimetre diameter has no disturbing effect whatever. Its paralyzing effects are so remarkable, that if we place a flat piece of iron or other metal across the maximum line of force, where loud sounds are given out, the instant that this flat piece is moved, so that one or both edges touch or end in the minimum line of force, it becomes instantly neutralized, giving out no sound whatever, notwithstanding that a large mass of metal lies in the maximum line of force. We will now demonstrate by experiment the exceeding sensitiveness of the induction-balance to the smallest piece of metal, if it is in the maximum line of force, and no part of it touching the minimum ; and also that by allowing either or both ends of this or a very large piece of metal to cut or end at the minimum lines of force, complete paralysis and consequent silence are produced *.

VI. There is a marked difference of the rapidity of action between all metals, silver having an intense rapidity of action. The induced currents from hard steel or from iron strongly magnetized are much more rapid than those from pure soft iron; the tones are at once recognized, the iron giving out a dull, heary smothered tone, whilst hard steel has tones exceedingly sharp. If we desire to balance iron, we can only balance it by a solid mass equal to the iron to be balanced. No amount of fine wires of iron can balance this mass, as the time of discharge of these wires is much quicker than that of a larger mass of iron. Hard steel, however, can be easily balanced not only by steel but by fine iron wires, and the degree of the fineness of these wires required to produce a balance gives a very fair estimate of the proportionate time of discharge. The rapidity of discharge has no direct relation with its electrical conductivity; for copper is much slower than zinc, and they are both superior to iron.

We find that the induction-balance is exceedingly sensitive to all molecular changes which take place in all metals subjected to any of the imponderable forces. Thus we have already by its aid studied the effects on metals of heat, magnetism, electricity, \&c., and of mechanical changes such as strain, torsion, and pressure ; and I propose in some future paper to describe the remarkable results already obtained, and to demonstrate by experiment today to the Physical Society the results of these forces upon the induction-balance.

* This was fully demonstrated to the audience by numerous experiments. 
Phil. Mag. S. 5.Vol 8. P1. V.

SONOMETER

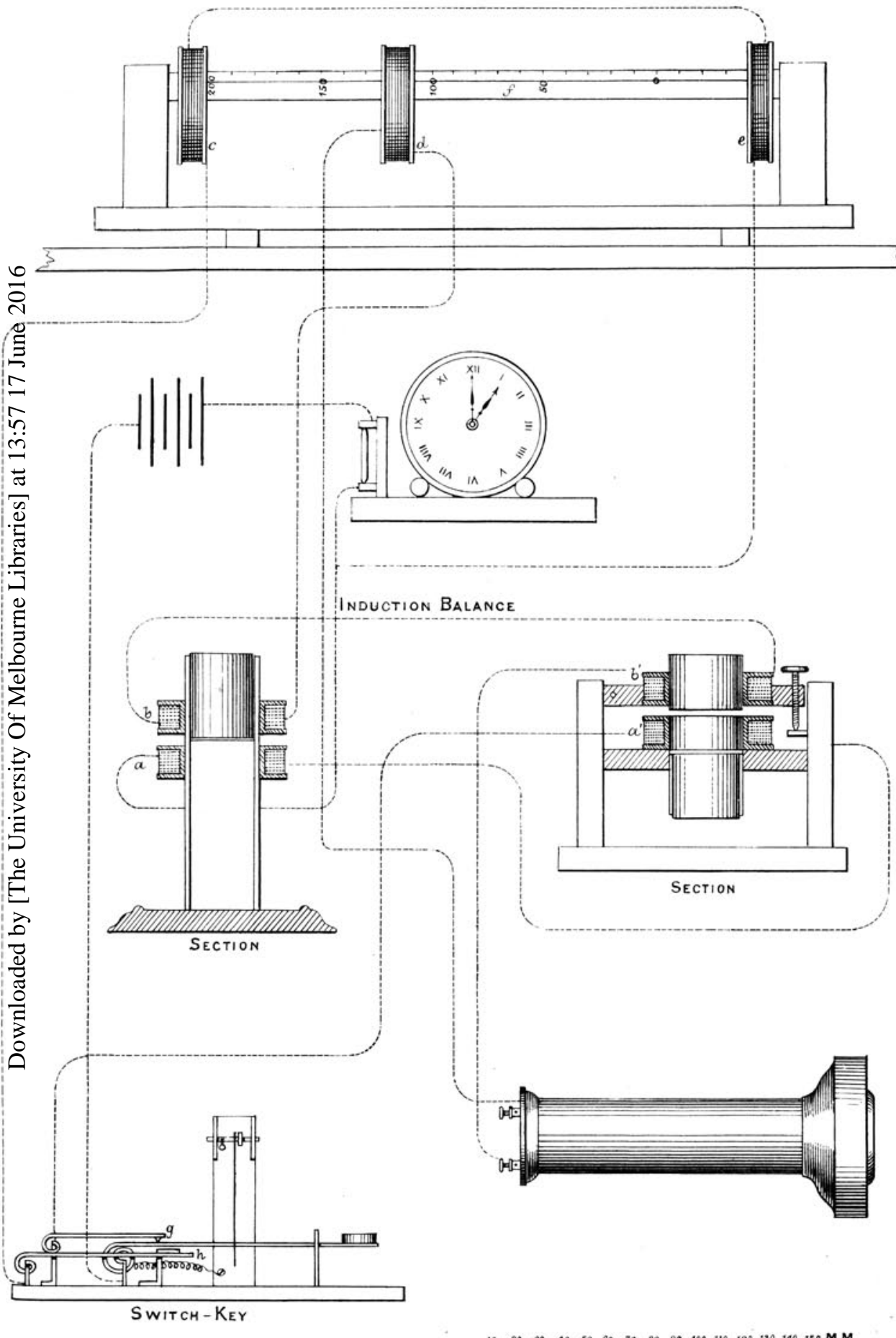

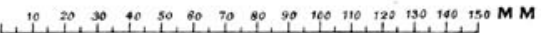

